\title{
High-resolution dispersion-based measurement of the electron beam energy spread
}

\author{
Eduard Prat $\odot,{ }^{1,}{ }^{*}$ Philipp Dijkstal $\odot,{ }^{1,2}$ Eugenio Ferrari, ${ }^{1}$ \\ Alexander Malyzhenkov, ${ }^{1}$ and Sven Reiche ${ }^{1}$ \\ ${ }^{1}$ Paul Scherrer Institut, CH-5232 Villigen PSI, Switzerland \\ ${ }^{2}$ ETH Zürich, Zürich 8093, Switzerland
}

(Received 15 July 2020; accepted 24 August 2020; published 2 September 2020)

\begin{abstract}
The energy spread of the electron beam is a critical parameter in x-ray free-electron lasers (XFELs) and needs to be optimized for best performance. The uncorrelated energy spread of the electrons can be a few $\mathrm{keV}$ or less in XFEL injectors, thus very challenging to measure. The standard method to characterize the electron beam energy spread, consisting in streaking the beam with a transverse deflector and measuring the time-resolved beam size of the electrons in a dispersive location for a single electron beam energy, has a typical resolution of several $\mathrm{keV}$. To overcome this limitation we introduce a novel method to measure the beam size at a dispersive location for different beam energies so that it is possible to disentangle the beam size contributions related to the energy spread, the intrinsic beam size and the monitor resolution. As a consequence, the energy spread can be characterized with a much higher precision and resolution than in the standard approach. We also suggest to perform measurements for different deflection amplitudes so that the energy spread induced by the transverse deflector can be subtracted properly. The scheme does not require any additional hardware and thus can be readily applied in any standard XFEL facility. Numerical simulations and experimental results at SwissFEL confirm the validity of our method. Our calculations show that the approach can be used to significantly overcome the resolution of the standard approach and measure energy spreads well below $1 \mathrm{keV}$. As an example we present energy spreads of few $\mathrm{keV}$ measured at the SwissFEL injector.
\end{abstract}

DOI: 10.1103/PhysRevAccelBeams.23.090701

\section{INTRODUCTION}

X-ray free-electron-lasers (XFELs) are state-of-the-art research instruments that have revolutionized science by enabling the observation of matter at atomic length and time scales [1-10]. The FEL process is driven by a highbrightness electron beam that travels through an undulator. The peak current, the emittance and the energy spread defining the electrons' brightness need to be measured and optimized for best FEL performance.

In particular, the energy spread of the electron beam is a fundamental parameter in XFEL facilities. First, the electron beam needs to have an uncorrelated relative energy spread at the undulator smaller than the so-called Pierce parameter [11], which for $\mathrm{x}$-rays typically varies between $10^{-4}$ and $10^{-3}$. In addition to this fundamental limit, the energy spread of the electron beam in general impacts the

\footnotetext{
*eduard.prat@psi.ch
}

Published by the American Physical Society under the terms of the Creative Commons Attribution 4.0 International license. Further distribution of this work must maintain attribution to the author(s) and the published article's title, journal citation, and DOI.
FEL performance: the lower the energy spread the lower the electrons' longitudinal velocity spread, thus the larger the radiation power and the shorter the saturation length. The initial energy spread is also a critical parameter for ultrashort pulse generation as it defines the final electron bunch length after full compression-the smaller the energy spread, the more the bunch can be compressed. Moreover, the energy spread is crucial for seeded FELs $[7,12]$, where it determines the amount of seed power needed for introducing the required energy modulation, e.g., in high-gain harmonic upshift FEL schemes used to access wavelengths much shorter than the seed wavelength $\lambda_{S}$ [13]. Such modulation in turn increases the final energy spread and limits the shortest achievable wavelength.

Radio frequency (rf) photoinjectors are normally used as sources in XFEL facilities to generate an electron beam with typical energies of 5-10 MeV, peak currents of 10-20 A, and energy spreads at the keV level or below. The beam is then accelerated and compressed to reach the $\mathrm{GeV}$ energies and $\mathrm{kA}$ peak currents required at the undulator entrance. The initial energy spread is increased at the undulator to the $\mathrm{MeV}$ level due to compression and because of potential detrimental effects like microbunching instability [14-17]. The small energy spread values of 
electron beams in XFEL facilities are very challenging to measure, specially at the injector, where the relative energy spread can be on the order of $10^{-5}$ or less.

The standard way to characterize the energy spread is by measuring the transverse beam size of the electron beam in a dispersive location, e.g., after a dipole magnet in a bunch compressor or spectrometer line (see for instance Refs. [18-20]). This approach has been proven to have resolutions down to a couple of $\mathrm{keV}$ [18]. An rf transverse deflector is normally used to streak the beam in the direction orthogonal to the dispersion. In this way, a measurement of the longitudinal phase space and of the uncorrelated energy spread becomes possible. The deflector, however, induces additional energy spread, which needs to be taken into account for high-resolution measurements. The energy spread can also be retrieved from undulator radiation measurements, for instance using optical replicas [21], via coherent harmonic generation [22], or exploiting the optical klystron effect [23]. Here we focus on the method using dispersion.

The energy spread is usually obtained assuming that the measured beam size is dominated by the dispersion; i.e., the relative energy spread is calculated as $\sigma_{E} / E=\sigma_{M} / D$, where $E$ is the mean energy of the electron beam, $\sigma_{M}$ is the measured rms beam size, and $D$ the dispersion. This approach, however, only provides an upper limit to the energy spread, since the contributions to the measured beam size coming from the intrinsic beam size and the profile monitor resolution are not considered (subtracted). For ultralow energy spreads, these contributions can be comparable or larger than the dispersive component, thus resulting in a large overestimation of the energy spread.

In this paper we introduce a new method to measure the energy spread with enhanced resolution. The basic idea is to measure the beam size for different beam energies in such a way that the effects coming from the intrinsic beam size and the profile monitor resolution can be adequately determined and accounted for. We also suggest, in order to assess the impact of the deflector, to perform measurements for different streaking amplitudes. This approach provides a much higher resolution than the standard approach. Moreover it also allows retrieving other relevant parameters such as the resolution of the profile monitor and the intrinsic beam size (useful to confirm the quality and optics of the beam). This method can be applied to any XFEL, since it only requires the possibility to measure the beam size in a dispersive location for different energies and deflector voltages, which is achievable in any standard facility.

The article is organized as follows. We will first describe the method. Later we will show how it works for a numerical example. Finally we will present experimental results at SwissFEL [10], an XFEL facility that recently started to operate at the Paul Scherrer Institute in Switzerland.

\section{DESCRIPTION OF THE METHOD}

In a dispersive location the transverse beam size has two contributions: the component due to dispersion and the intrinsic beam size contribution, which for relativistic beams can be approximated as $D \sigma_{E} / E$ and $\sqrt{m_{e} c^{2} \beta \varepsilon_{n} / E}$, respectively, where $E$ is the beam mean energy, $\beta$ is the $\beta$-function at the profile monitor, $\varepsilon_{n}$ the normalized emittance, and $m_{e} c^{2}$ the rest-mass energy of an electron $(0.511 \mathrm{MeV})$, with $m_{e}$ being the electron mass and $c$ the speed of light. In addition to the dispersive and intrinsic beam size components, the observed beam size will be affected by the profile monitor resolution $\sigma_{R}$, which we assume to be independent on the energy. We also assume that the dispersion component of the beam size, the intrinsic contribution and the monitor resolution are uncorrelated so they add quadratically to the measured beam size $\sigma_{M}$ :

$$
\sigma_{M}^{2}=\sigma_{R}^{2}+\frac{m_{e} c^{2} \beta \varepsilon_{n}}{E}+\frac{D^{2} \sigma_{E}^{2}}{E^{2}} .
$$

\section{A. Standard approach}

The energy spread is normally derived directly from the measured beam size and the dispersion: $\sigma_{E}=\sigma_{M} E / D$. This approximation ignores effects related to intrinsic beam size and profile monitor resolution. Thus, it may result in a significant overestimation of the retrieved energy spread. The resolution of the energy spread measurement with this approach (i.e., the minimum measurable value) can be expressed as:

$$
R_{\sigma_{E}}=\frac{E}{D} \sqrt{\sigma_{R}^{2}+\frac{m_{e} c^{2} \beta \varepsilon_{n}}{E}}
$$

To improve the resolution, several measures can be taken. First, the measurement should be done at low energy, but still avoiding the regime where space-charge forces are significant [24]. Second, the profile monitor resolution should be as small as possible. Third, the measurement optics should be optimized to have a large dispersion and a small $\beta$-function at the monitor location.

The standard approach can be refined by making assumptions on the contributions of the intrinsic beam size or resolution (and subtract them from the measured beam size). These assumptions, however, may have large uncertainties, especially concerning the resolution, which is normally not known beforehand.

\section{B. New approach}

In our approach we improve the measurement resolution by appropriately taking into account the contributions from the intrinsic beam size and the profile monitor resolution. The idea is to measure the square of the beam size for different beam energies $E$ and fit the results according to Eq. (1). The method exploits the different 


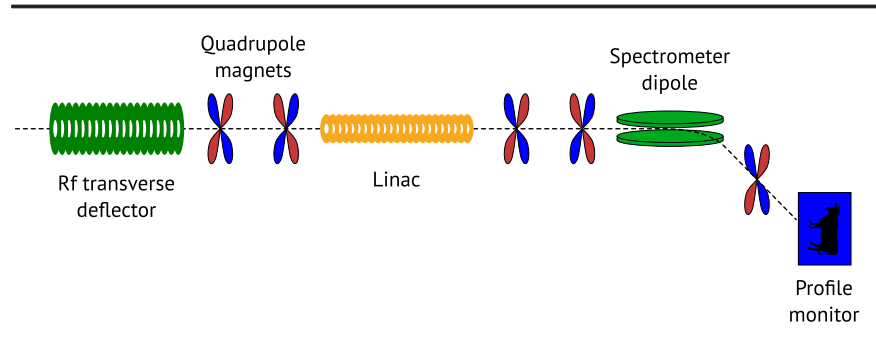

FIG. 1. Sketch of the measurement setup.

energy dependences of the three components of the measured beam size: the monitor resolution is assumed to be independent on the energy, the intrinsic beam size scales with $1 / E$ and the dispersive contribution depends on $1 / E^{2}$. The required energy change can be achieved by adjusting the voltage or the phase of rf structures before the spectrometer and scaling the magnets accordingly. This approach works assuming that the $\beta$-function, the normalized emittance, the dispersion, and the absolute energy spread of the beam do not change with energy. This is ensured in the absence of space-charge forces and by scaling the optics with the energy variation. The method has the additional advantage that it allows reconstructing the monitor resolution and the product of emittance and $\beta$-function.

The quality of the energy spread reconstruction depends on the weight of each of the three contributions to the measured beam size. The errors of the reconstructed energy spread will be larger when the beam size is dominated by the profile monitor resolution and intrinsic beam size components, and smaller the more the dispersive component contributes to the measured beam size. To increase the dispersive contribution and therefore to reduce the uncertainty of the energy spread reconstruction, the same prescriptions as the ones to improve the resolution of the standard approach [see Eq. (2)] apply: perform the measurement at low energies, maximize the dispersion, minimize the monitor resolution, and minimize the $\beta$-function.

Figure 1 shows a sketch of the measurement setup. First, the beam is streaked with an rf transverse deflector. A linac is used to vary the energy for the measurements. Here the linac is located after the deflector, but the positions of the two may just as well be inverted. The beam size measurements are performed with a profile monitor placed in a dispersive location. Several quadrupoles are used to optimize the optics. In particular, a quadrupole after the dipole can be used to enlarge the dispersion value at the monitor. Standard XFEL facilities are equipped with all the required components to implement this measurement scheme.

\section{Transverse deflector effects}

When using a deflector, the uncorrelated energy spread induced by the deflector can be calculated as [25]:
$\sigma_{E_{T}}=e k V \cos (\phi) \sigma_{T}=e k V \cos (\phi) \sqrt{\frac{m_{e} c^{2} \beta_{T} \varepsilon_{n_{T}}}{E_{T}}}$

where $e$ is the elementary charge, $k$ is the wave number of the deflector, $V$ is the deflector voltage, $\phi$ is the operating phase of the deflector (normally the deflector operates at the zero crossing so $\cos (\phi)=1$ ), and $\sigma_{T}$ is the average transverse beam size in the structure, which can be obtained from the $\beta$-function, the emittance in the streaking plane and the energy at the deflector (respectively $\beta_{T}, \varepsilon_{n_{T}}$, and $\left.E_{T}\right)$. For slice energy spread measurements, the deflector voltage should be chosen to have an optimum balance between small induced energy spread and enough streaking to overcome the rf curvature effects.

The energy spread induced by the deflector adds quadratically to the energy spread of the beam $\sigma_{E 0}$ :

$$
\begin{aligned}
\sigma_{E}^{2} & =\sigma_{E 0}^{2}+\sigma_{E_{T}}^{2}=\sigma_{E 0}^{2}+e^{2} k^{2} V^{2} \cos ^{2}(\phi) \sigma_{T}^{2} \\
& =\sigma_{E 0}^{2}+e^{2} k^{2} V^{2} \cos ^{2}(\phi) \frac{m_{e} c^{2} \beta_{T} \varepsilon_{n_{T}}}{E_{T}}
\end{aligned}
$$

The deflector contribution can be subtracted if the parameters determining it are known. A better alternative is to perform the full characterization described above (beam size measurements for different energies) for different deflector voltages. With such a two-dimensional scan, both the deflector contribution and the true energy spread can be reconstructed from Eqs. (1) and (4).

There are two possibilities, depending on the deflector's location with respect to the linac used for the energy change. If the deflector is placed before the linac, as displayed in Fig. 1 and realized in SwissFEL, the energy at the deflector is constant, and the reconstructed energy spread at the linac entrance includes the energy spread before the deflector and the induced energy spread by the deflector. In this case, one could perform the full energy scan for different deflector voltages to obtain $\sigma_{E}$ as a function of $V$ at the linac entrance. This data can then be fitted according to Eq. (4) to reconstruct both the energy spread before the deflector and the deflector contribution.

If the deflector is located after the linac, we have $E_{T}=E$ and the energy at the deflector changing during a full measurement of $\sigma_{E}$. Consequently, the beam size at the deflector $\sigma$ and the induced energy spread also varies with the energy change. We can rewrite Eq. (1) replacing $\sigma_{E}$ by Eq. (4) and using $E_{T}=E$ :

$$
\begin{aligned}
\sigma_{M}^{2}= & \sigma_{R}^{2}+\frac{m_{e} c^{2} \beta \varepsilon_{n}}{E}+\frac{D^{2} \sigma_{E 0}^{2}}{E^{2}} \\
& +\frac{D^{2} e^{2} k^{2} V^{2} \cos ^{2}(\phi) m_{e} c^{2} \beta_{T} \varepsilon_{n_{T}}}{E^{3}} .
\end{aligned}
$$

We see that, for this situation, the beam size acquires an additional term proportional to $1 / E^{3}$. In this case, one 
would perform beam size measurements for different energies and deflector voltages and fit the data with the above equation.

\section{NUMERICAL CALCULATIONS}

We have validated our method with numerical calculations. We take an example consistent with uncompressed bunches at an XFEL facility, with typical pulse durations of several picoseconds, for which the absolute energy spread is the lowest. We consider a profile resolution of $15 \mu \mathrm{m}$, a normalized emittance of $150 \mathrm{~nm}$, and the optics used at the SwissFEL injector for this measurement: a $\beta$-function of $0.7 \mathrm{~m}$ and a dispersion of $1.5 \mathrm{~m}$ at the profile monitor. Table I shows the parameters used in the simulations. We will first evaluate the reconstruction of the energy spread without a deflector. After that we will analyze how to retrieve the energy spread considering the effects of a deflector placed before the linac used for the energy change. Finally, we will evaluate the impact of the most important systematic errors.

\section{A. Reconstruction of the energy spread, the monitor resolution, and the intrinsic beam size contribution}

We have calculated the implementation of the method based on Eq. (1) for the above-mentioned parameters. In each simulated measurement the energy is scanned between 100 and $400 \mathrm{MeV}$ (we expect that space-charge effects are negligible for these energies). For our parameters and the lowest energy of $100 \mathrm{MeV}$, the resolution of the standard measurement approach is $1.84 \mathrm{keV}$ (see Eq. (2). We consider different energy spread values between 0.25 and $5 \mathrm{keV}$ (in steps of $0.25 \mathrm{keV}$ ). For each energy spread case we keep the same conditions shown in Table I. The beam sizes as a function of the energy are fitted according to Eq. (1). For the fit, we apply a nonlinear least squares regression using a trust-region algorithm. When fitting, the beam size data are weighted according to their uncertainties. We force the resolution, the intrinsic beam size and the dispersive contributions to be larger than zero (as negative values would not be physical). We consider 10 shots for each beam size measurement, adding a statistical random error of $2.5 \%$ to the true beam sizes in each shot (this error magnitude is consistent with our observations at SwissFEL).

Figure 2 shows the calculated parameters for the different cases. The graphs show average and standard deviation

TABLE I. Simulation parameters.

\begin{tabular}{lc}
\hline \hline Parameter & Value \\
\hline Profile monitor resolution & $15 \mu \mathrm{m}$ \\
Normalized emittance & $150 \mathrm{~nm}$ \\
$\beta$-function & $0.7 \mathrm{~m}$ \\
Dispersion & $1.5 \mathrm{~m}$ \\
\hline \hline
\end{tabular}
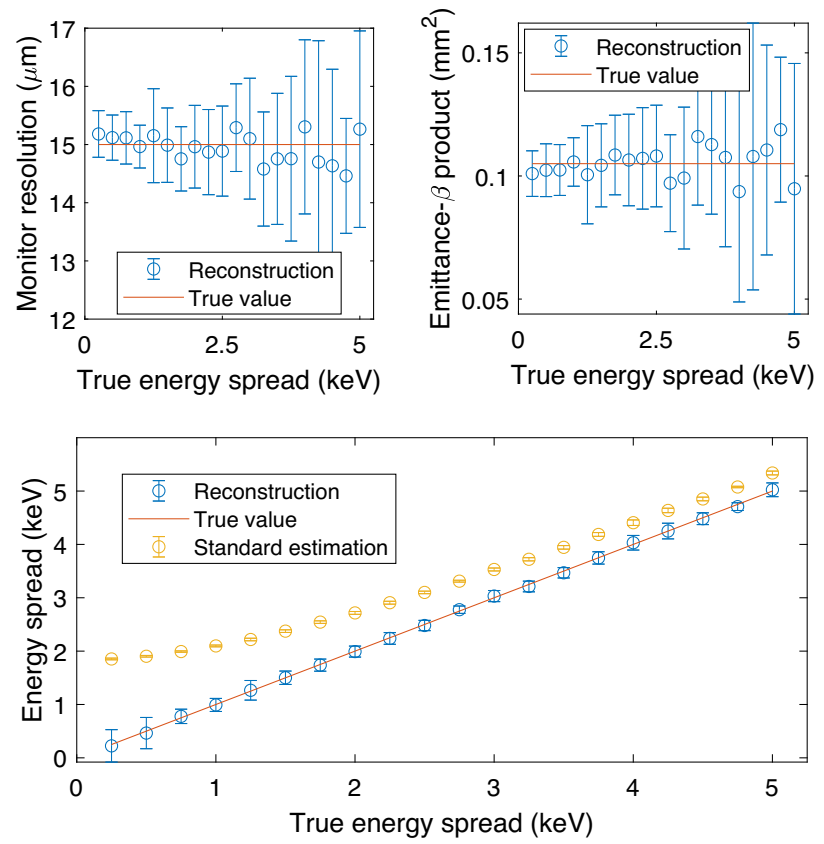

FIG. 2. Calculated performance of the method for the parameters shown in Table I and energy spread values between 0.25 and $5 \mathrm{keV}$. We show the true value and reconstruction of the monitor resolution (top left), intrinsic beam size (top right) and energy spread (bottom). The energy spread estimation using the standard approach is also displayed.

for the reconstructed parameters over ten calculations. We also display the energy spread that would be obtained with the standard approach (i.e., $E \sigma_{M} / D$ ). We observe that the three contributions to the beam size measurement are adequately derived. While the standard approach would be quite close to the true value for large energy spreads, a large overestimation would result for small energy spreads - the standard estimation tends to the measurement resolution of $1.84 \mathrm{keV}$ for small energy spread values. We also see that the uncertainty of the reconstructed energy spread increases for small energy spreads. This is because, for small values, the contribution of the energy spread to the beam size is relatively small and thus more difficult to extract from the fit. Similarly, the uncertainties for the profile monitor and beam size components increase when these contributions are less significant, i.e., for larger energy spread values. Figure 3 shows a calculation example for an energy spread of $1.5 \mathrm{keV}$. We plot the measured beam size as a function of the energy and the fit results for each of the contributions.

\section{B. Impact of rf transverse deflector}

We consider the situation where the deflector is placed before the linac, as it is realized in SwissFEL and also depicted in Fig. 1. In this situation, the reconstructed energy spread at the linac entrance includes the energy spread before the deflector and the energy spread induced by the 


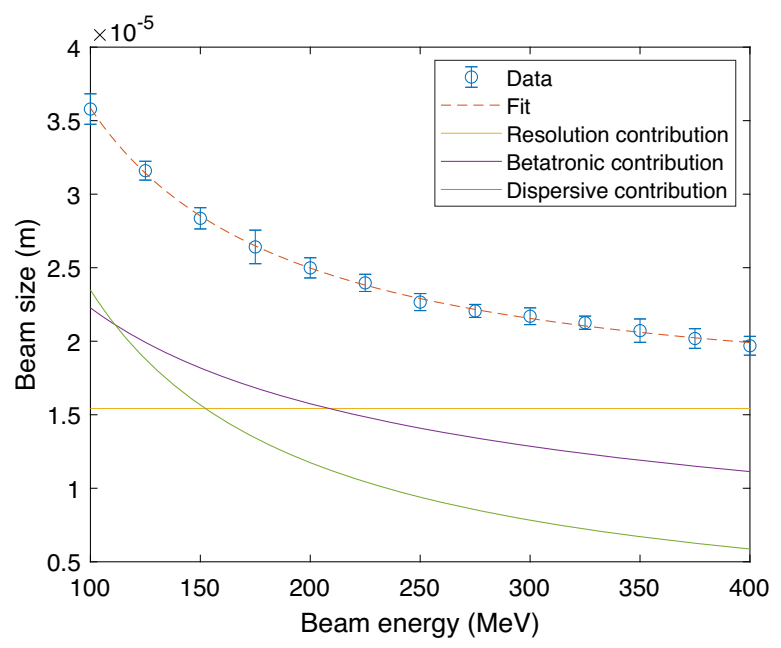

FIG. 3. Beam sizes at the profile monitor and the obtained fits for the different contributions for a particular example of the results of Fig. 2 with an energy spread of $1.5 \mathrm{keV}$.

deflector. As described in the previous section, to reconstruct the initial energy spread and the deflector contribution we would perform energy scans for different deflector voltages.

We have calculated the implementation of the method for the parameters defined before and an energy spread of $1 \mathrm{keV}$ at the entrance of the deflector. The voltage of the deflector $V$ is varied between 0.2 and $0.6 \mathrm{MV}$ in steps of $0.1 \mathrm{MV}$, the $k$ value is $60 \mathrm{~m}^{-1}$ (approximate value corresponding to the standard S-band frequency used at the SwissFEL injector), the energy at the deflector is $E_{T}=300 \mathrm{MeV}$, and the $\beta$-function at the deflector is $\beta_{T}=50 \mathrm{~m}$, giving a beam size of $113 \mu \mathrm{m}$ for a normalized emittance of $150 \mathrm{~nm}$. The energy spread induced by the deflector $\sigma_{E_{T}}$, calculated with Eq. (3), varies between $1.36 \mathrm{keV}$ (for $V=0.2 \mathrm{MV}$ ) and $4.07 \mathrm{keV}$ (for $V=0.6 \mathrm{MV}$ ). We choose a minimum voltage of $0.2 \mathrm{MV}$ because this gives a sufficient streaking for the measurement of uncompressed bunches at SwissFEL. For each deflector voltage the energy spread at the linac entrance is reconstructed as explained before. After that, a fit according to Eq. (4) is

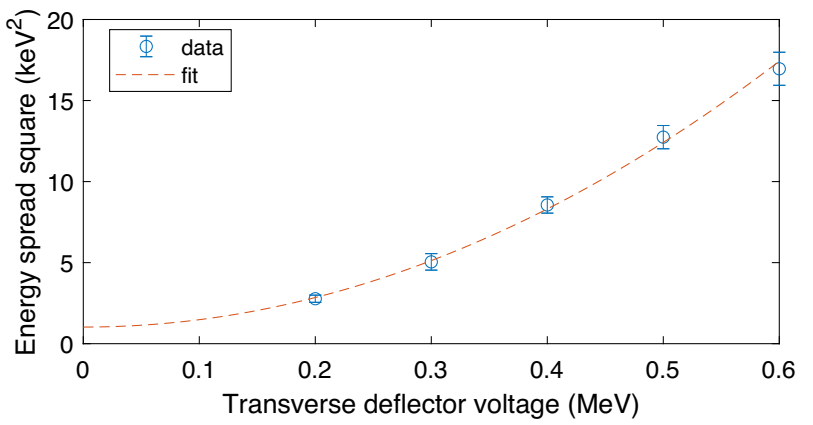

FIG. 4. Square of the reconstructed energy spread as a function of the deflector voltage for a case in which the initial energy spread is $1 \mathrm{keV}$. applied (we use the same fit method and algorithm as described earlier).

Figure 4 shows an example of the square of the reconstructed energy spread at the linac entrance as a function of the deflector voltage, together with the applied fit. From ten calculations we obtain a reconstructed energy spread before the deflector of $0.95 \pm 0.10 \mathrm{keV}$, and a deflector contribution $k \sigma_{T}$ of $6.80 \pm 0.18 \times 10^{-3}$ (the true value is $6.78 \times 10^{-3}$ ). The reconstructed energy spread ignoring the deflector contribution, i.e., the measured value for the lowest deflector voltage, would be $1.7 \mathrm{keV}$, overestimating the true value by $70 \%$.

\section{Systematic errors}

The most important systematic errors in the reconstruction of the energy spread are due to the uncertainties of the beam size measurement, the energy, and the dispersion. The beam size measurement error may come from the profile monitor calibration or from errors in determining the rms size from the transverse profile measurement (e.g., uncertainties associated to applying Gaussian fits to obtain the beam size). The beam size, energy and dispersion errors will affect the value of the reconstructed energy spread according to Eq. (1). In case the deflector is used and its effects are considered, the deflector voltage uncertainty may also affect the measurement. In this subsection, however, we will not consider such effects.

We have calculated the impact of the systematic errors related to the beam size, energy, and dispersion. We have considered the same parameters described before and an energy spread of $2 \mathrm{keV}$, with relative errors varying between $\pm 10 \%$. On top of each systematic error we also consider, as before, a statistical beam size measurement error of $2.5 \%$. Figure 5 shows the results of the energy

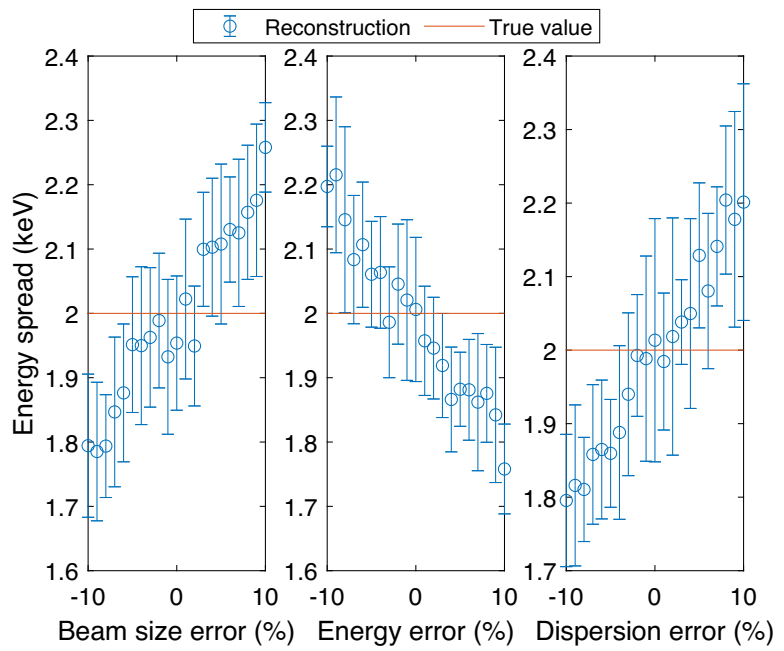

FIG. 5. Reconstructed energy spread as a function of different systematic relative errors: beam size (left), energy (center), and dispersion (right). 
spread reconstruction as a function of different error amplitudes. Each point shows the result (average and standard deviation) over 10 calculations. We can observe that the three systematic errors have a roughly linear impact on the reconstruction: a $10 \%$ error approximately corresponds to a $10 \%$ error in the reconstruction, which for this particular case is comparable to the statistical errors due to beam size variation. Considering that in a typical FEL facility the beam size, the energy and the dispersion are known to a few percent or better, we can conclude that these systematic errors will impact the measurement of the energy spread also at the few-percent level or less.

\section{MEASUREMENTS}

Subjecting our method to an experimental test we have measured the energy spread of the electron beam core at the SwissFEL injector. Figure 1 shows a sketch of the experimental setup. The electron beam is accelerated with the S-band injector to the design energy of $300 \mathrm{MeV}$ at the entrance of the deflector. We streak the beam vertically with an S-band transverse deflector [26], while the spectrometer dipole deflects in the horizontal plane. The beam energy is changed with the first two modules of the SwissFEL C-band linac, located between the deflector and the spectrometer. The optics are the same as the ones considered for the numerical calculations: a $\beta$-function of $0.7 \mathrm{~m}$ and a dispersion of $1.5 \mathrm{~m}$ at the profile monitor. The beam size is measured with a Ce:YAG scintillator screen [27] placed at the injector spectrometer.

\section{A. Results}

We performed measurements for two setups: one for the standard configuration for uncompressed bunches with a bunch charge of $200 \mathrm{pC}$ and an rms pulse duration of $3 \mathrm{ps}$, and one with the same conditions but for a charge of $10 \mathrm{pC}$. The final beam energy was varied between 100 and $430 \mathrm{MeV}$. Within this energy range we expect no significant space-charge effects. We took five beam size measurements at each energy value. For stability reasons, the energy variation was accomplished by varying the phases of the rf modules at a fixed maximum voltage (around $250 \mathrm{MV}$ for each module). The sum of the phases was fixed at 180 degrees, such that no additional linear energy chirp was added to the bunch. The quadrupoles and dipoles within and after the C-band linac were scaled according to the energy change to ensure the same optics and dispersion for the full measurement range.

Figure 6 shows single-shot images of the streaked beam at the spectrometer and the corresponding slice beam sizes for the $10 \mathrm{pC}$ case and different energies. We divide the extent of the beam in the streaked direction in several slices (31 in the example of the figure), and apply Gaussian fits to determine beam size and centroid for each slice. The observed differences in the slice centroid curves (i.e., the slice energy along the bunch) for the different images are explained by the phase changes of the C-band linac during the energy scan. The orf curvature gives a varying energy chirp along the slices which results into an increase of the observed beam sizes in the dispersive direction. We determine the beam size only for the core slice, for which the rf curvature effect is minimal. The core slice is defined as the one in the center part having the maximum (or minimum) position, i.e., energy. We have checked the consistency of our results for different numbers of slices: the slice beam size for the core does not significantly vary when the beam is divided into 21,31 , or 41 slices. In the following we report the results obtained 31 slices.
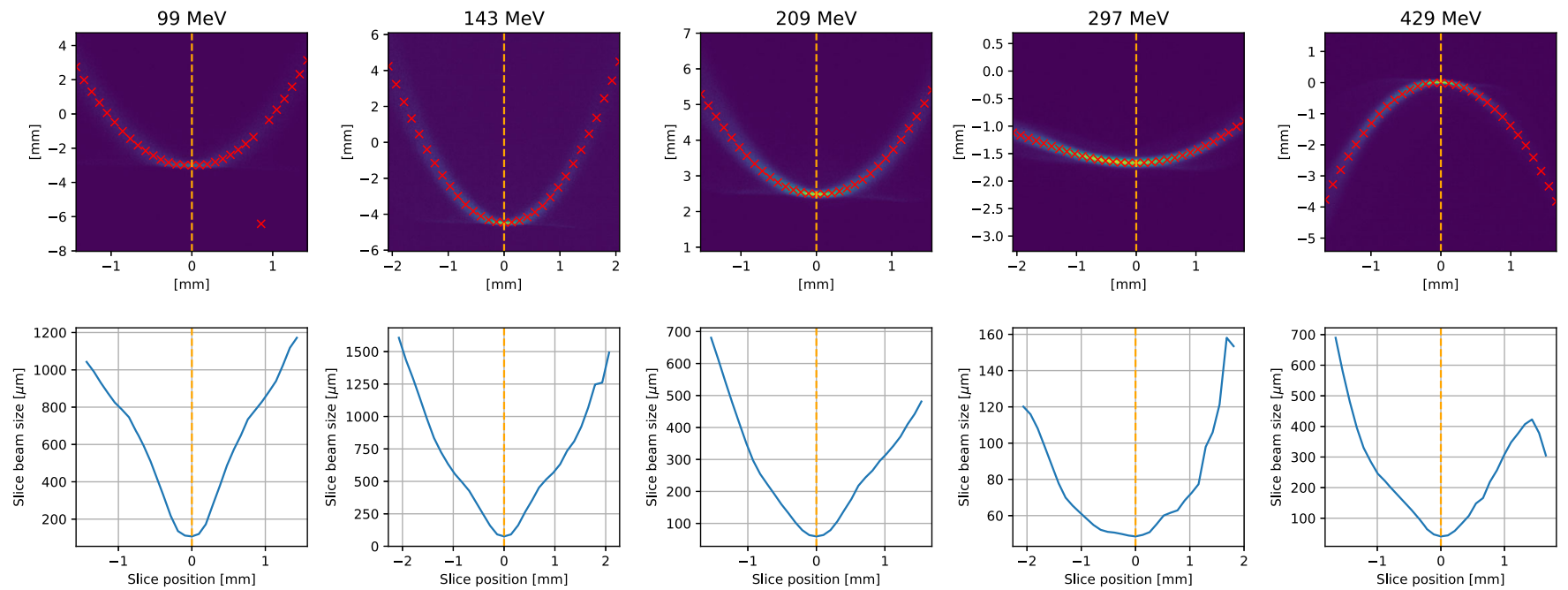

FIG. 6. Beam images (top) and corresponding slice beam sizes (bottom) for the $10 \mathrm{pC}$ measurement and different energies. The beam is vertically streaked and horizontally dispersed, but to align the images with the slice beam size curves beneath, we have rotated the images such that the streaking direction appears as horizontal and the dispersive direction as vertical. The red crosses indicate the slice centroids. The core slice horizontal beam size is marked by the orange dashed line. 


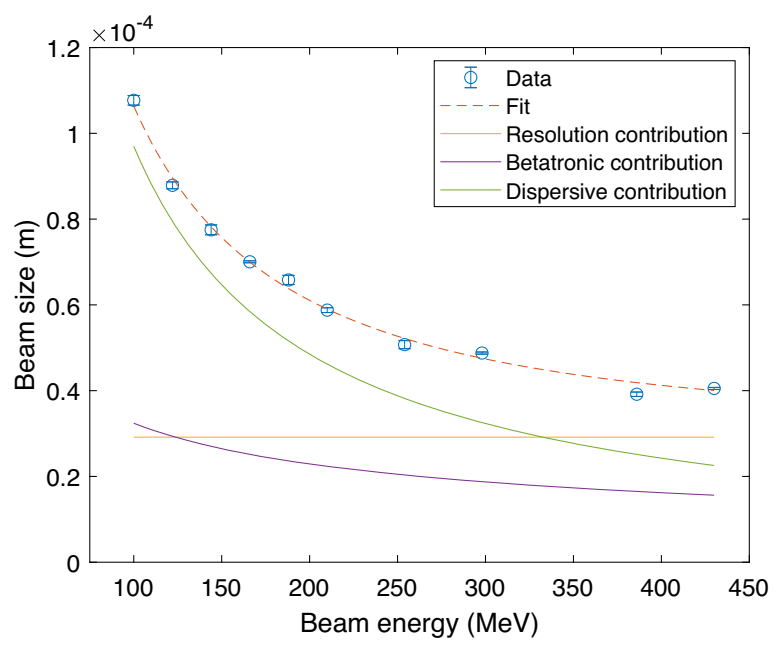

FIG. 7. Measured beam sizes at the profile monitor and corresponding fits for the different contributions for a beam charge of $10 \mathrm{pC}$. The reconstructed energy spread is $6.5 \mathrm{keV}$.

Once the beam size is measured for the different energies, we fit the results using Eq. (1), as explained before. The results for $200 \mathrm{pC}$ are the following: the energy spread is $15.0 \pm 0.3 \mathrm{keV}$, the monitor resolution is $33 \pm 8 \mu \mathrm{m}$, and the intrinsic beam size contribution is practically zero. For $10 \mathrm{pC}$ charge, we obtain an energy spread of $6.5 \pm 0.5 \mathrm{keV}$, a profile monitor resolution of $29 \pm 6 \mu \mathrm{m}$, and an emittance- $\beta$ product of $0.2 \pm 0.3 \mathrm{~mm}^{2}$. Figure 7 shows the measured beam sizes as a function of energy for the $10 \mathrm{pC}$ case, together with the corresponding fits. We confirmed the reproducibility of the method by repeating the measurement at the $200 \mathrm{pC}$ conditions, obtaining equivalent results between the two measurements. The dispersive component dominates the beam size for both bunch charges, more strongly in the $200 \mathrm{pC}$ case. The profile monitor resolution is consistently reconstructed to be around $30 \mu \mathrm{m}$, matching with the results obtained in [28] for a different monitor. The intrinsic beam size contribution turns out to be the least important. Because of this, the corresponding relative error is also the largest.

\section{B. Discussion}

\section{Comparison with the standard approach}

The resolution of the standard approach [see Eq. (2)] for an energy of $100 \mathrm{MeV}$, with the design optics, the retrieved profile monitor resolution of about $30 \mu \mathrm{m}$, and a normalized slice emittance of 150 (60) nm for 200 (10) pC (consistent with the emittance results presented in [28]), would be $2.5 \mathrm{keV}$ for $200 \mathrm{pC}$ and $2.2 \mathrm{keV}$ for $10 \mathrm{pC}$. The resolution is mostly dominated by the profile monitor resolution-assuming a zero-emittance beam, the resolution would still be $2 \mathrm{keV}$. Since the resolution of the standard method is rather small compared to the measured energy spreads, the results we get with our method do not differ much from those obtained by dividing the measured beam size (at the lowest energy) by the dispersion: for $200 \mathrm{pC}$, the direct measurement would give $15.1 \pm 0.6 \mathrm{keV}$, equivalent to the result from our method; for the $10 \mathrm{pC}$ measurement, the standard approach would give an energy spread of $7.1 \pm 0.1 \mathrm{keV}$, about $10 \%$ larger than the value found with our method. Still, in the standard approach one could not distinguish which part of the retrieved energy spread is due to resolution issues, whereas our method provides more assurance that the measured energy spread is not affected by resolution.

\section{Transverse deflector effects}

The reported measurements were done for a deflector voltage of $0.4 \mathrm{MV}$. According to Eq. (3), and taking a $\beta$-function at the structure of $50 \mathrm{~m}$ (corresponding to the design optics), and a normalized emittance of 150 (60) nm for 200 (10) $\mathrm{pC}$, this corresponds to an induced energy spread of $2.7 \mathrm{keV}$ for $200 \mathrm{pC}$ and $1.7 \mathrm{keV}$ for the $10 \mathrm{pC}$ case. These values are relatively small in comparison to the measured energy spreads. Subtracting the deflector contribution from the measured values of $15.0 \mathrm{keV}$ for $200 \mathrm{pC}$ and $6.5 \mathrm{keV}$ for $10 \mathrm{pC}$ [see Eq. (4)], we would arrive at true energy spreads of $14.8 \mathrm{keV}$ for $200 \mathrm{pC}$ and $6.3 \mathrm{keV}$ for $10 \mathrm{pC}$. Due to the limited beam time available we did not perform additional measurements for different deflector voltages to confirm the small contribution of the deflector to the reconstructed energy spreads.

\section{Comparison with simulations and other experimental observations}

The experimentally determined energy spreads at the SwissFEL injector are significantly larger than expected. Simulation results produced with ASTRA [29,30] predict an uncorrelated energy spread before compression well below $1 \mathrm{keV}$. These simulations include space-charge but do not consider intrabeam scattering [31,32] or cathode effects. The large measured energy spreads could be attributed to intrabeam scattering or to the fact that SwissFEL uses cesium telluride cathodes (which have a longer emission time than the standard copper material used in most other FEL facilities). In the future, systematic measurements may be performed to investigate these two effects that could explain the relatively large observed energy spread values.

The large energy spreads found in our measurements are, however, consistent with a number of other experimental observations: First, the energy spread values determined at the injector match well with measurements performed further downstream, after compression. We have measured an energy spread of 750-1000 keV for beams with peak currents of 1.2-1.5 kA (these measurements were done by simply dividing the beam size by the dispersion, but here we expect no significant contributions from intrinsic beam size, monitor resolution or deflector). Assuming that the 
longitudinal emittance is preserved, this corresponds to 10-17 keV for our initial current of approximately $20 \mathrm{~A}$, fitting well with our measurements at the injector. Second, the use of a laser heater, a device commonly used to suppress microbunching in XFEL facilities by increasing the energy spread $[17,19,33,34]$, so far has not helped significantly to increase the FEL pulse energy at SwissFEL. This observation is compatible with or could be explained by the relatively large energy spread values reported here. Third, SwissFEL tends to deliver its best FEL performance when operating at comparatively low peak currents (1.5-2 kA), consistent with a rather large initial energy spread (a higher peak current increases the relative energy spread at the undulator to values larger than the Pierce parameter, thereby degrading the FEL performance).

\section{CONCLUSION}

We have presented a novel, precise and high-resolution method to characterize the energy spread of electron beams. In contrast to the standard approach, in which the beam size is measured in a dispersive location at a single energy, we perform several measurements at different energies. In this way, the contributions related to the intrinsic beam size and the profile monitor resolution can be inferred and, as a consequence, the energy spread can be reconstructed with higher resolution. We also suggest to assess the contribution of the deflector by performing measurements at different deflector voltages. The scheme can be readily implemented at any XFEL facility without any additional hardware or cost. We have shown by way of a numerical example that the approach can be used to resolve energy spreads as small as $1 \mathrm{keV}$ and below, hence significantly improving on the resolution of the standard procedure. The method has been implemented experimentally at SwissFEL, where we have measured energy spreads down to $6 \mathrm{keV}$.

\section{ACKNOWLEDGMENTS}

We thank Thomas Schietinger for improving the language of the manuscript. We acknowledge all the technical groups involved in the operation of SwissFEL. This work has been supported by the SNF Grant No. 200021175498.

[1] Z. Huang and K-J. Kim, Review of X-ray free-electron laser theory, Phys. Rev. Accel. Beams 10, 034801 (2007).

[2] B. W. J. McNeil and N. R. Thompson, X-ray free-electron lasers, Nat. Photonics 4, 814 (2010).

[3] C. Pellegrini, A. Marinelli, and S. Reiche, The physics of X-ray free-electron lasers, Rev. Mod. Phys. 88, 015006 (2016).

[4] W. Ackermann et al., Operation of a free-electron laser from the extreme ultraviolet to the water window, Nat. Photonics 1, 336 (2007).
[5] P. Emma et al., First lasing and operation of an ångstromwavelength free-electron laser, Nat. Photonics 4, 641 (2010).

[6] T. Ishikawa et al., A compact X-ray free-electron laser emitting in the sub-ångström region, Nat. Photonics 6, 540 (2012).

[7] E. Allaria et al., Two-stage seeded soft-X-ray free-electron laser, Nat. Photonics 7, 913 (2013).

[8] H.-S. Kang et al., Hard X-ray free-electron laser with femtosecond-scale timing jitter, Nat. Photonics 11, 708 (2017).

[9] W. Decking et al., A MHz-repetition-rate hard X-ray freeelectron laser driven by a superconducting linear accelerator, Nat. Photonics 14, 391 (2020).

[10] C. Milne et al., SwissFEL: The swiss x-ray free electron laser, Appl. Sciences 7, 720 (2017).

[11] R. Bonifacio, C. Pellegrini, and L. M. Narducci, Collective instabilities and high-gain regime in a free electron laser, Opt. Commun. 50, 373 (1984).

[12] P. R. Ribič et al., Coherent soft X-ray pulses from an echoenabled harmonic generation free-electron laser, Nat. Photonics 13, 555 (2019).

[13] L.-H. Yu, Generation of intense uv radiation by subharmonically seeded single-pass free-electron lasers, Phys. Rev. A 44, 5178 (1991).

[14] S. Heifets, G. Stupakov, and S. Krinsky, Coherent synchrotron radiation instability in a bunch compressor, Phys. Rev. Accel. Beams 5, 064401 (2002).

[15] Z. Huang and K.-J. Kim, Formulas for coherent synchrotron radiation microbunching in a bunch compressor chicane, Phys. Rev. Accel. Beams 5, 074401 (2002).

[16] E. L. Saldin, E. A. Schneidmiller, and M. V. Yurkov, Klystron instability of a relativistic electron beam in a bunch compressor, Nucl. Instrum. Methods Phys. Res., Sect. A 490, 1 (2002).

[17] E. L. Saldin, E. A. Schneidmiller, and M. V. Yurkov, Longitudinal space charge-driven microbunching instability in the TESLA Test Facility linac, Nucl. Instrum. Methods Phys. Res., Sect. A 528, 355 (2004).

[18] M. Hüning and H. Schlarb, Measurement of the beam energy spread in the TTF photo-injector, in Proceedings of the 20th Particle Accelerator Conference, Portland, OR, 2003 (IEEE, New York, 2003), p. 2074.

[19] Z. Huang et al., Measurements of the linac coherent light source laser heater and its impact on the $\mathrm{x}$-ray free-electron laser performance, Phys. Rev. Accel. Beams 13, 020703 (2010).

[20] J. H. Lee, J.-H. Han, S. Lee, J. Hong, C. H. Kim, C. K. Min, and I. S. Ko, PAL-XFEL laser heater commissioning, Nucl. Instrum. Methods Phys. Res., Sect. A 843, 39 (2017).

[21] E. L. Saldin, E. A. Schneidmiller, and M. V. Yurkov, A simple method for the determination of the structure of ultrashort relativistic electron bunches, Nucl. Instrum. Methods Phys. Res., Sect. A 539, 499 (2005).

[22] C. Feng, T. Zhang, J. Chen, H. Deng, M. Zhang, X. Wang, B. Liu, T. Lan, D. Wang, and Z. Zhao, Measurement of the average local energy spread of electron beam via coherent harmonic generation, Phys. Rev. Accel. Beams 14, 090701 (2011). 
[23] E. Prat, E. Ferrari, S Reiche, and T. Schietinger, Using the optical-klystron effect to increase and measure the intrinsic beam energy spread in free-electron-laser facilities, Phys. Rev. Accel. Beams 20, 040702 (2017).

[24] M. Ferrario, M. Migliorati, and L. Palumbo, Space Charge Effects, Contribution to the CERN Accelerator School: Advanced Accelerator Physics Course, Trondheim, Norway (CERN, Geneva, 2013).

[25] K. Floettmann and V. V. Paramonov, Beam dynamics in transverse deflecting rf structures, Phys. Rev. Accel. Beams 17, 024001 (2014).

[26] P. Craievich et al., Transverse deflecting structures for bunch length and slice emittance measurements on SwissFEL, in Proceedings of the 35th International FreeElectron Laser Conference, New York, USA, 2013, (JACoW, Geneva, 2013), 236.

[27] R. Ischebeck, E. Prat, V. Thominet, and C. O. Loch, Transverse profile imager for ultrabright electron beams, Phys. Rev. Accel. Beams 18, 082802 (2015).

[28] E. Prat et al., Generation and Characterization of Intense Ultralow-Emittance Electron Beams for Compact
X-Ray Free-Electron Lasers, Phys. Rev. Lett. 123, 234801 (2019).

[29] K. Floettmann, ASTRA, A Space Charge Tracking Algorithm, http://www.desy.de/mpyflo/Astra_dokumentation/, 2000.

[30] K. Floettmann, Note on the thermal emittance of electrons emitted by Cesium Telluride photo cathodes TESLA-FEL Report No. 1997-01.

[31] A. Piwinski, Intrabeam Scattering, in CERN Accelerator School, 226 (1991), https://doi.org/10.5170/CERN-1992001.226.

[32] Z. Huang, Intrabeam Scattering in an x-ray FEL driver, Reports No. LCLS-TN-02-8 and No. SLAC-TN-05-026, 2002.

[33] Z. Huang, M. Borland, P. Emma, J. Wu, C. Limborg, G. Stupakov, and J. Welch, Suppression of microbunching instability in the linac coherent light source, Phys. Rev. Accel. Beams 7, 074401 (2004).

[34] S. Spampinati et al., Laser heater commissioning at an externally seeded free-electron laser, Phys. Rev. Accel. Beams 17, 120705 (2014). 